\title{
Paper
}

\section{Thermal and Mechanical Properties of Harmonic-Structured Composite with Copper and Molybdenum}

\author{
Hiroshi FUJIWARA ${ }^{1 *}$, Koki SONE ${ }^{2}$, Fuminori UEGUCHI ${ }^{3}$ and Hiroyuki MIYAMOTO ${ }^{4}$ \\ ${ }^{1}$ Department of Mechanical Engineering, Shizuoka Institute of Science and Technology, 2200-2 Toyosawa, Fukuroi 437-8555, Japan. \\ ${ }^{2}$ Undergraduate Student, Shizuoka Institute of Science and Technology, 2200-2 Toyosawa, Fukuroi 437-8555, Japan. \\ ${ }^{3}$ Graduate Student, Doshisha University, 1-3 Miyakodani, Tatara, Kyotanabe 610-0394, Japan. \\ ${ }^{4}$ Department of Mechanical and Systems Engineering, Doshisha University, 1-3 Miyakodani, Tatara, Kyotanabe 610-0394, Japan.
}

Received December 9, 2017; Revised January 7, 2018; Accepted January 11, 2018

\begin{abstract}
$\mathrm{Cu}-\mathrm{Mo}$ harmonic-structured composite was produced via mechanical milling (MM) followed by spark plasma sintering (SPS), and its thermal and mechanical properties were investigated in detail. Microstructural observation of the MM powders and SPS compacts was achieved using scanning electron microscopy (SEM)/energy dispersive X-ray spectroscopy (EDS). The mechanical properties of the SPS compacts were evaluated using results of the Vickers hardness test. The thermal properties of them were evaluated using results of thermo-mechanical analysis and laser flash method. SEM/EDS micrographs profile indicated that the $\mathrm{Cu}-\mathrm{Mo}$ harmonic-structured composite has the network region composed of molybdenum and the dispersed region of copper. The $\mathrm{Cu}-\mathrm{Mo}$ harmonicstructured composites demonstrate low coefficient of linear expansion and enough thermal conductivity compared to the conventional $\mathrm{Cu}-\mathrm{Mo}$ particle dispersed composite. The hardness of $\mathrm{Cu}-\mathrm{Mo}$ harmonic-structured composite corresponds to the Voigt model of the rule of mixture of $\mathrm{Cu}$ and Mo. The harmonic structure control is effective for an improvement of the thermal and mechanical properties.
\end{abstract}

\section{KEY WORDS}

harmonic structure, coefficient of linear expansion, thermal conductivity, mechanical milling, spark plasma sintering

\section{Introduction}

In recent years, integrated circuits (IC) have been miniaturized with the miniaturization of electronic devices. The amount of heat generated by the high-density and high-power output has increased than ever before. Therefore, a heat spreader with high thermal conductivity effectively move the heat from a heat source ${ }^{1)}$. The thermal properties of both low coefficient of thermal expansion (C.T.E.) and high thermal conductivity are favored as the heat spreader materials, because the IC mainly consists of a silicon with low C.T.E. A copper is suitable for heat spreader materials for the high thermal conductivity in metals, but the C.T.E. is larger than that of the semiconductors. The large difference of C.T.E. causes a separation between semiconductor and heat spreader for a cyclic thermal stress. On the other hand, the C.T.E. of molybdenum is close to that of the silicon and the molybdenum has higher thermal conductivity than the silicon. A Cu-Mo composite with the low C.T.E. and high thermal conductivity comparatively is suitable as the heat spreader materials. However, in $\mathrm{Cu}-\mathrm{Mo}$ composite, increase of the $\mathrm{Cu}$ rate induces high C.T.E. and high thermal

* Corresponding author, E-mail: fujiwara.hiroshi@sist.ac.jp

** The content of this article had been presented at JSPMIC2017. conductivity. On the contrary, increase of the Mo rate induces low C.T.E. and low thermal conductivity. Therefore, it is difficult to introduce the thermal properties of the low C.T.E and high thermal conductivity simultaneously in the $\mathrm{Cu}-\mathrm{Mo}$ composite materials. The low C.T.E and high thermal conductivity is tradeoff relationship in $\mathrm{Cu}-\mathrm{Mo}$ composite. Whereas, the harmonicstructured materials simultaneously demonstrate two mechanical properties in the trade-off relationship, for example of the high strength and high ductility ${ }^{2}$. The harmonic structure has a possibility that suppressing the trade-off relationship can be introduced to the $\mathrm{Cu}-\mathrm{Mo}$ composite. However, the research on the thermal properties of harmonic-structured materials have not yet. In this paper, the $\mathrm{Cu}-$ Mo harmonic-structured composite is produced via the mechanical milling (MM) followed by the spark plasma sintering (SPS), and its thermal and mechanical properties are investigated in detail.

\section{Experimental}

Pure copper powder (99.7 mass\% purity; Nippon Atomized Metal Powders Corporation) and pure molybdenum powder (99.9 mass\% purity; Kojundo Chemical Laboratory Co., Ltd.) were used in this study. The initial particle sizes of the pure $\mathrm{Cu}$ and the pure Mo 
powders were approximately 80 and $3.0 \mu \mathrm{m}$, respectively. These powders were mechanically milled using a planetary ball mill (P-6; Fritch Co., Ltd.) with an SKD11 vessel and SUJ2 steel balls in Ar atmosphere at room temperature. Mixed powder consisting of pure Mo and pure $\mathrm{Cu}$ (50.0 to 80.0 mass\%) was mechanically milled at a ball-to-powder weight ratio of 1:2, an MM time of 86.4 to $172.8 \mathrm{ks}$ and a rotation speed of 200 and $250 \mathrm{rpm}$. The mechanically milled powder containing pure $\mathrm{Cu}$ and Mo was sintered using an SPS apparatus (SPS-510L; Sumitomo Coal Mining Co., Ltd.) at $1173 \mathrm{~K}$ and $100 \mathrm{MPa}$ for $1.8 \mathrm{ks}$. Scanning electron microscopy (SEM)/ energy dispersive X-ray spectroscopy (EDS) were employed to observe the microstructure of the SPS compact. The mechanical properties of the SPS compacts were evaluated by the Vickers hardness test (VMT-7; Matsuzawa Co., Ltd.) using an indenter with the load of $196.1 \mathrm{~N}$. The thermal properties of them were evaluated by the thermo-mechanical analysis (TMA: SS6100, NIS nano technology Co., Ltd.) at temperature elevation rate of $0.17 \mathrm{~K} / \mathrm{s}$ under Ar atmosphere and laser flash method (LDA447 Nanoflash, NEtzsch Co., Ltd.).

\section{Results and discussion}

The MM powder in which pure $\mathrm{Cu}$ and pure Mo powders were combined by MM for 172.8 ks is shown in Fig. 1. Figs. 1 (a) and (b) show SEM micrographs of the appearance and cross section of the MM powder with $\mathrm{Cu}$ (70.0 mass\%), respectively. Figs. 1 (c) and (d) indicate EDS results for the $\mathrm{Cu}$ and Mo concentrations in the cross section shown in Fig. 1 (b), respectively. The MM powder shown in Fig. 1 (b) exhibits a different contrast in the surface and inner regions, and Figs. 1 (c) and (d) demonstrate that the surface region has high Mo concentration. The shell/core powder as shown in Fig. 1 was sintered using an SPS apparatus, and an interesting microstructure was obtained. Figs. 2 (a), (b), (c) and (d) show this microstructure in SEM micrographs of the SPS compacts of the shell/core powders with the $\mathrm{Cu}$ in the mass fraction of $80,70,60$ and $50 \%$, respectively. Relative densities for each SPS compact are also shown in Fig. 2. Each SPS compact has an enough sintered density for the relative density of approximately $99 \%$. These micrographs have a network structure with Mo material shown by a bright contrast, and its network region corresponds to the surface region of the MM powder shown in Fig. 1. The network width increases with decreasing the mass fraction of $\mathrm{Cu}$. The dispersed region surrounding the network corresponds to $\mathrm{Cu}$ material. A microstructure having network and dispersed regions is referred to as a "harmonic structure."

Fig. 3 demonstrates coefficient of linear expansion at $373 \mathrm{~K}$ to $1073 \mathrm{~K}$ of the harmonic-structured composites with 50.0 to 80.0 mass\% $\mathrm{Cu}$ and only $\mathrm{Cu}$ and Mo SPS compact. The changes in the coefficient of linear expansion from $323 \mathrm{~K}$ to $1073 \mathrm{~K}$ of the harmonic-structured composites with less than 70.0 mass $\% \mathrm{Cu}$ are very small compared to that with 80.0 mass $\% \mathrm{Cu}$. Fig. 4 shows
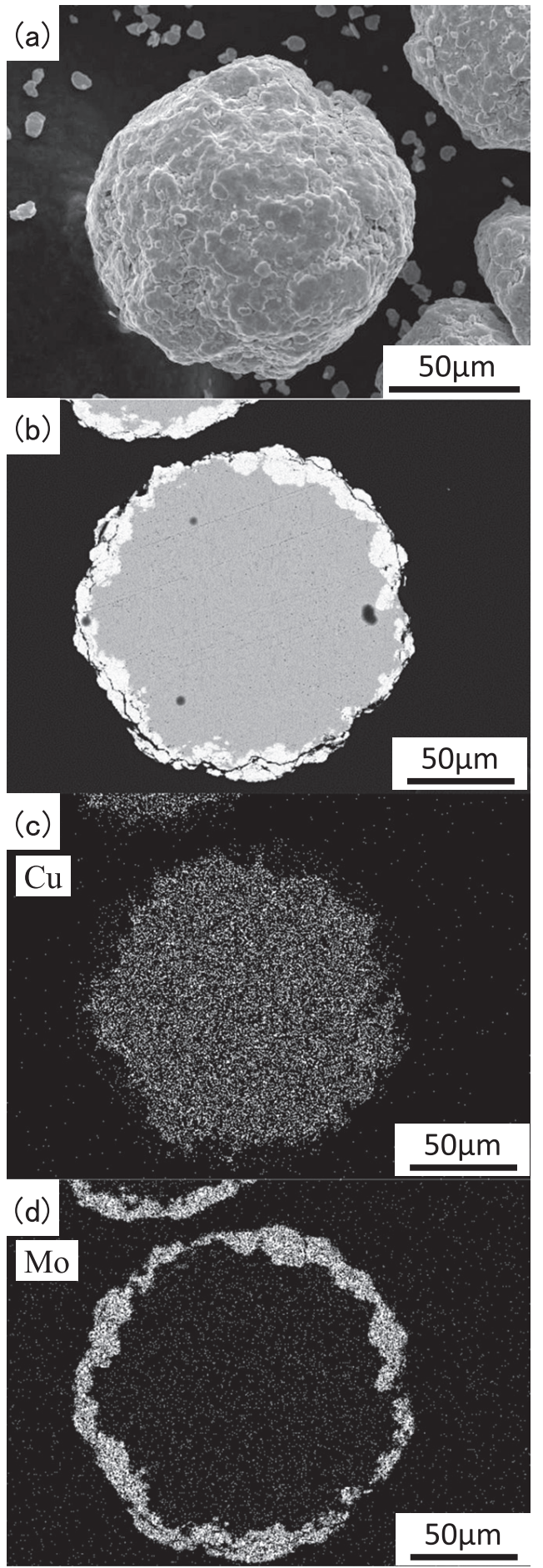

Fig. 1 SEM micrographs of the appearance (a) and the cross section (b) and the EDS elemental mapping results for $\mathrm{Cu}$ (c) and $\mathrm{Mo}$ (d) of the MM powder with $\mathrm{Cu}$ mass fraction of $70.0 \%$.

coefficient of linear expansion of the harmonic-structured and particle dispersed composite ${ }^{3)}$ with 70.0 mass $\% \mathrm{Cu}$. The particle dispersed composite is conventional $\mathrm{Cu}-\mathrm{Mo}$ composite consisting of $\mathrm{Cu}$ matrix and dispersed Mo particles ${ }^{1,4)}$. The coefficient of linear expansion of the particle dispersed composite increases with increasing temperature. On the other hand, the coefficient of linear expansion of the harmonic structured composite does not change 


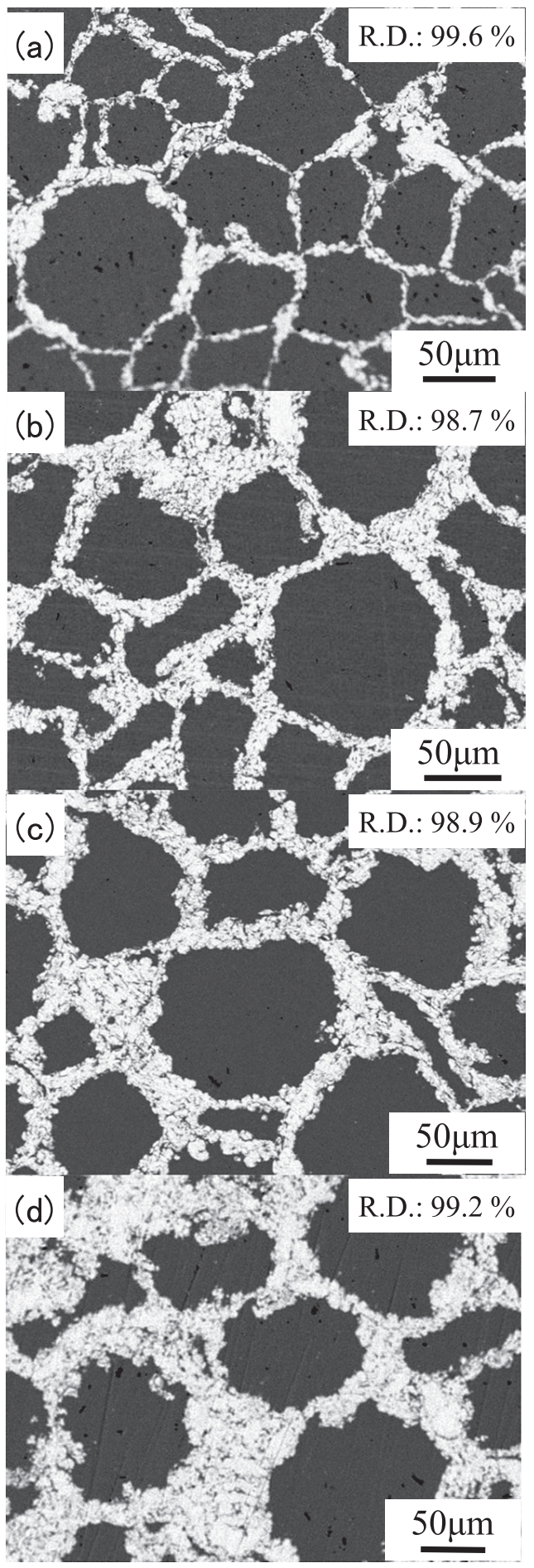

Fig. 2 SEM micrographs of the harmonic-structured composites sintered by SPS from the MM powders with the Cu mass fraction of 80.0 (a), 70.0 (b), 60.0 (c) and $50.0 \%(d)$.

even if temperature increase. Fig. 5 demonstrates the coefficient of linear expansion of the harmonic-structured and conventional particle dispersed composite ${ }^{4)}$ in the change of $\mathrm{Cu}$ mass fraction at $423 \mathrm{~K}$, which is a temperature comparable for the literature value. Each $\mathrm{Cu}-\mathrm{Mo}$ composite decreases with decreasing $\mathrm{Cu}$ mass fraction. However, the harmonic-structured composite has the lower coefficient of linear expansion than particle dispersed

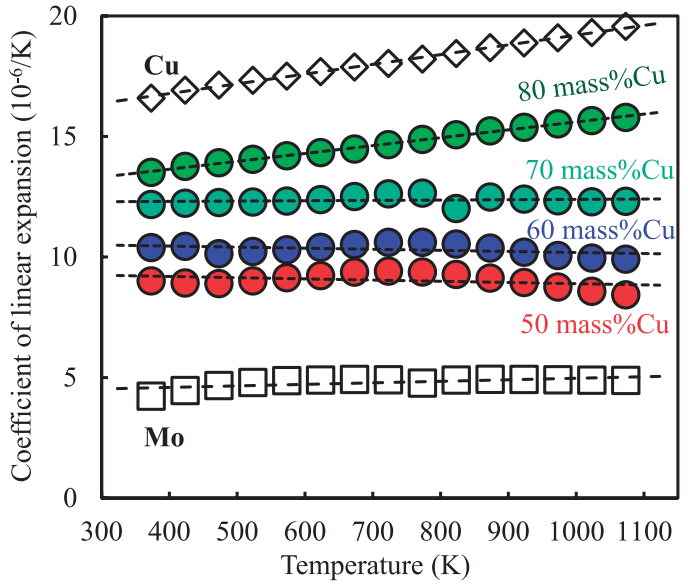

Fig. 3 Coefficient of linear expansion of the harmonic-structured composites with the $\mathrm{Cu}$ mass fraction of 50.0 to $80.0 \%$ at 373 to $1073 \mathrm{~K}$.

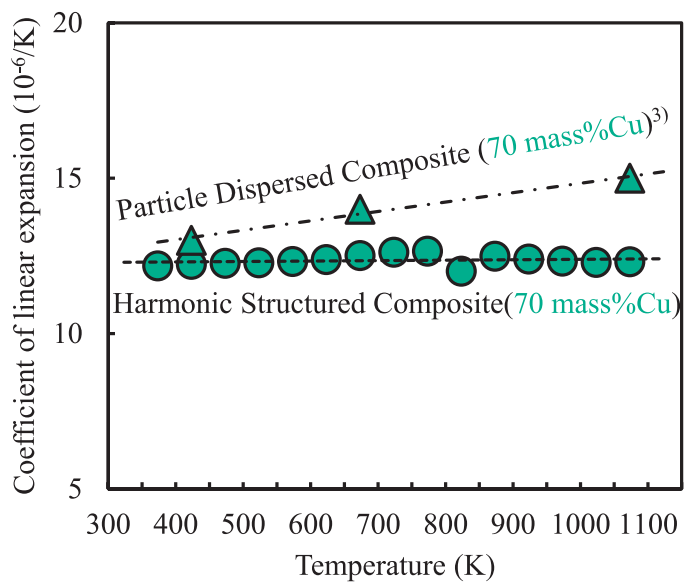

Fig. 4 Coefficient of linear expansion of the harmonic-structured and the particle dispersed composites with the $\mathrm{Cu}$ mass fraction of $70.0 \%$ at 373 to $1073 \mathrm{~K}$.

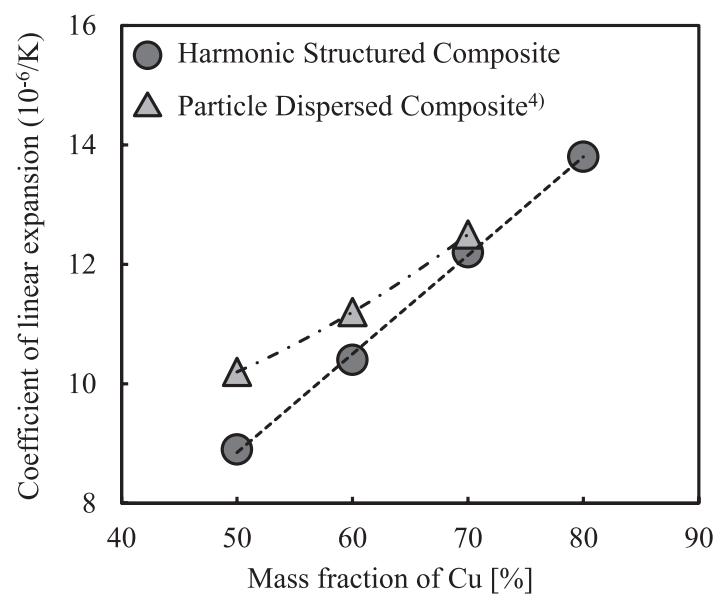

Fig. 5 Coefficient of linear expansion of the harmonic-structured and the particle dispersed composites with the $\mathrm{Cu}$ mass fraction of 50.0 to $80.0 \%$ at $423 \mathrm{~K}$.

composite in low $\mathrm{Cu}$ mass fraction area. These results reveal that harmonic-structured composite has excellent coefficient of linear expansion compared to conventional particle dispersed composite. 


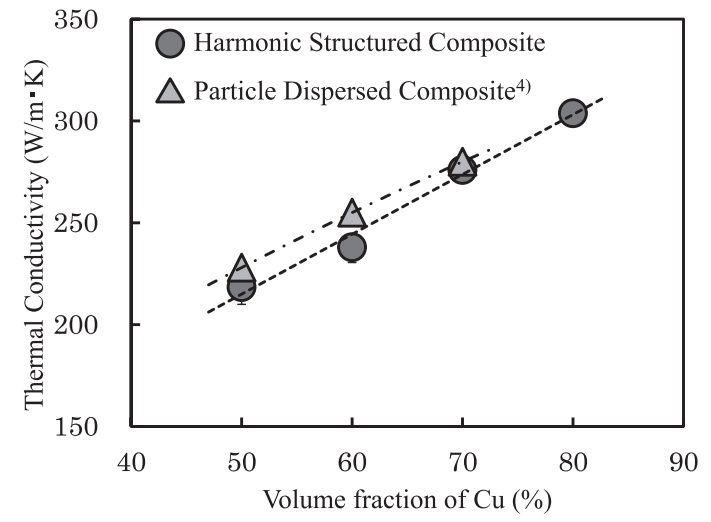

Fig. 6 Thermal conductivity of the harmonic-structured and conventional particle dispersed composites with the $\mathrm{Cu}$ mass fraction of 50.0 to $80.0 \%$.

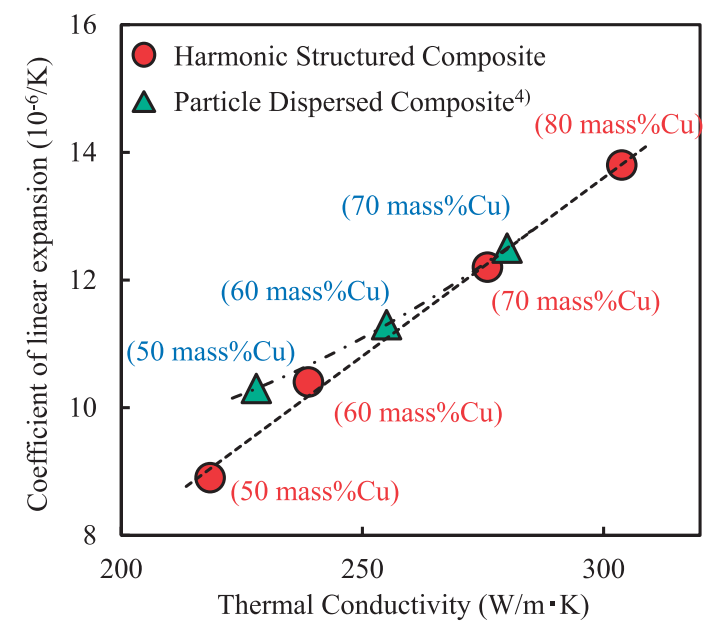

Fig. 7 Relationship between thermal conductivity and coefficient of linear expansion in the harmonic-structured and particle dispersed composites with the $\mathrm{Cu}$ mass fraction of 50.0 to $80.0 \%$.

The low coefficient of linear expansion of the harmonic-structured composite is attributed to the restriction for the thermal expansion of $\mathrm{Cu}$ by the network structure of Mo.

Fig. 6 shows the thermal conductivity of the harmonic-structured and conventional particle dispersed composite ${ }^{4)}$ in the change of $\mathrm{Cu}$ mass fraction. The thermal conductivity of the harmonicstructured composite is slightly lower than the particle dispersed composite. However the difference in the thermal conductivity between the harmonic-structured and particle dispersed composite is quite small. It is less than $7 \%$. Decrease of thermal conductivity of the harmonic-structured composite is attributed that the $\mathrm{Cu}$ area with high thermal conductivity is not continuously connected in the harmonic structure. Fig. 7 demonstrates the relationship between thermal conductivity and coefficient of linear expansion in the harmonic-structured and particle dispersed composite ${ }^{4)}$. Even though the harmonic-structured composite with 60.0 mass $\% \mathrm{Cu}$ and the particle dispersed composite with 50.0 mass $\% \mathrm{Cu}$ have almost the same coefficient of linear expansion, the thermal conductivity of the harmonic-structured composite is higher than that of the

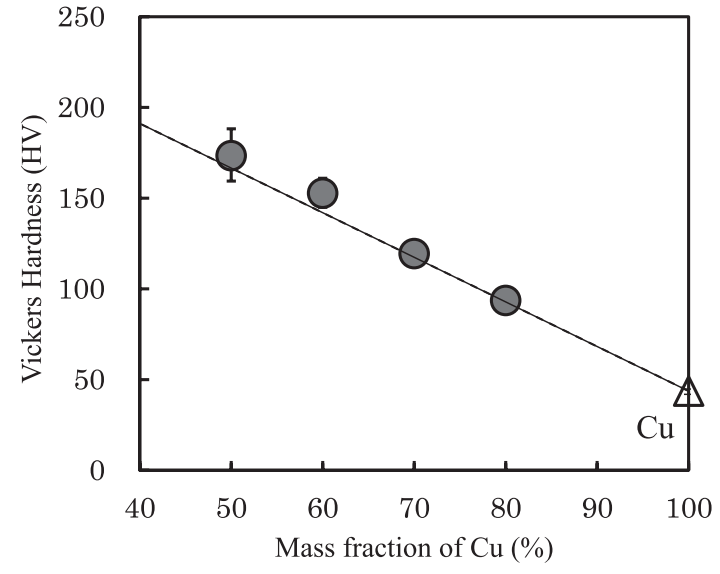

Fig. 8 Vickers hardness of the harmonic-structured composites with the $\mathrm{Cu}$ mass fraction of 50.0 to $80.0 \%$.

particle dispersed composite. Therefore, in the case of $\mathrm{Cu}-\mathrm{Mo}$ composite, harmonic structure can improve the thermal properties with the trade-off relationship between thermal conductivity and coefficient of linear expansion.

Fig. 8 shows the relationship between the Vickers hardness and $\mathrm{Cu}$ mass fraction. The Vickers hardness of the harmonic-structured composites with 50.0 to 80.0 mass $\% \mathrm{Cu}$ is corresponds to the value of Voigt model of the rule of mixture. In general, the hardness of the particle dispersed composites is lower than the Voigt model and higher than Reuss model of the rule of mixture ${ }^{5}$. High hardness of the harmonic-structured composites is attributed to the restriction by the network structure of Mo with high rigidness.

\section{Conclusion}

A harmonic-structured $\mathrm{Cu}-\mathrm{Mo}$ composite with a network region of pure $\mathrm{Mo}$ and a dispersed region of pure $\mathrm{Cu}$ was fabricated by a mechanical milling and spark plasma sintering process. Thermal and mechanical properties of the harmonic-structured $\mathrm{Cu}-\mathrm{Mo}$ composite were subsequently evaluated. The combined powder with a surface of pure Mo and an inner region of pure $\mathrm{Cu}$ was produced by the mechanical milling process. As this combined powder underwent spark plasma sintering, the harmonic-structured $\mathrm{Cu}$-Mo composite with a network region of pure Mo and a dispersed region of pure $\mathrm{Cu}$ was fabricated. The harmonic-structured $\mathrm{Cu}-$ Mo composite demonstrates high thermal conductivity in spite of almost the same coefficient of linear expansion compared to the conventional particle dispersed $\mathrm{Cu}-\mathrm{Mo}$ composite. The hardness of the harmonic-structured $\mathrm{Cu}-\mathrm{Mo}$ composite corresponds to the Voigt model of the rule of mixture of $\mathrm{Cu}$ and Mo.

\section{Acknowledgments}

This work is supported by the grant from Iketani Science and Technology Foundation (contact No. 0291031-A) and the support is gratefully appreciated. 


\section{References}

1) K. Matsubara, H. Kuroki, N. Sawai, Y. Takahara: J. Jpn Inst. Metals, 73 (2009) 211-215.

2) H. Fujiwara, T. Kawabata, H. Miyamoto, K. Ameyama: Mater. Trans., 54 (2013) 1619-1623.

3) Ametek, Molybdenum-Copper Composite AMC 3169, MatWeb, (reference Dec. 8, 2017). http://www.matweb.com/index.aspx
4) T. Arikawa, E. Takegoshi, Y. Hirasawa: Netsu Bussei, 11 (1997) 129-135.

5) H. S. Kim, S. I. Hong, S. J. Kim: J. Mater. Proces. Tech., 112 (2001) 109-113. 